\title{
The Big Bang and the genetic code
}

\author{
Gamow, a prankster and physicist , thought of them first
}

\section{Gino Segrè}

$\mathrm{T}$ he Big Bang and the genetic code are probably the two scientific ideas that have most radically altered our view of the world in the twentieth century. The Big Bang seeks to explain how the Universe was created and how the primordial constituents came to be formed. The genetic code lays down the pattern for the formation of living material and the transmission of inherited characteristics.

Interestingly enough, the first serious theories of both these key ideas were formulated by the same man, a colourful, irresistibly playful physicist named George Gamow. He was born in Odessa in 1904, began his education there and then moved to St Petersburg. In 1928, Gamow demonstrated that nuclear alpha decay could be understood by quantum mechanical tunnelling. He also showed that inverse tunnelling could occur, that is, sufficiently energetic protons would penetrate the nuclear barrier; this stimulated Cockroft and Walton to build the first machine capable of artificially disintegrating nuclei. So began, in 1932, the world of experimental nuclear physics.

A 1940 paper by Gamow and Mario Schoenberg was the first in a subject we now call particle astrophysics. The two authors presciently speculated that neutrinos could play a role in the cooling of massive collapsing stars. They named the neutrino reaction the Urca process, after a well known Rio de Janeiro casino. This name might seem a strange choice, but not to Gamow, a legendary prankster who once submitted a paper to Nature in which he suggested that the Coriolis force might account for his observation that cows chewed clockwise in the Northern Hemisphere and counterclockwise in the Southern Hemisphere.

In the 1940s Gamow began to attack, with his colleague Ralph Alpher, the problem of the origin of the chemical elements. Their first paper on the subject appeared in a 1948 issue of the Physical Review. At the last minute Gamow, liking the sound of 'alpha, beta, gamma', added his old friend Hans Bethe as middle author in absentia (Bethe went along with the joke, but the editors did not). Gamow and Alpher, with Robert Herman, then pursued the idea of an extremely hot neutron-dominated environment. They envisioned the neutrons decaying into protons, electrons and anti-neutrinos and, when the universe had cooled sufficiently, the neutrons and protons assembling heavier nuclei. They even estimated the photon

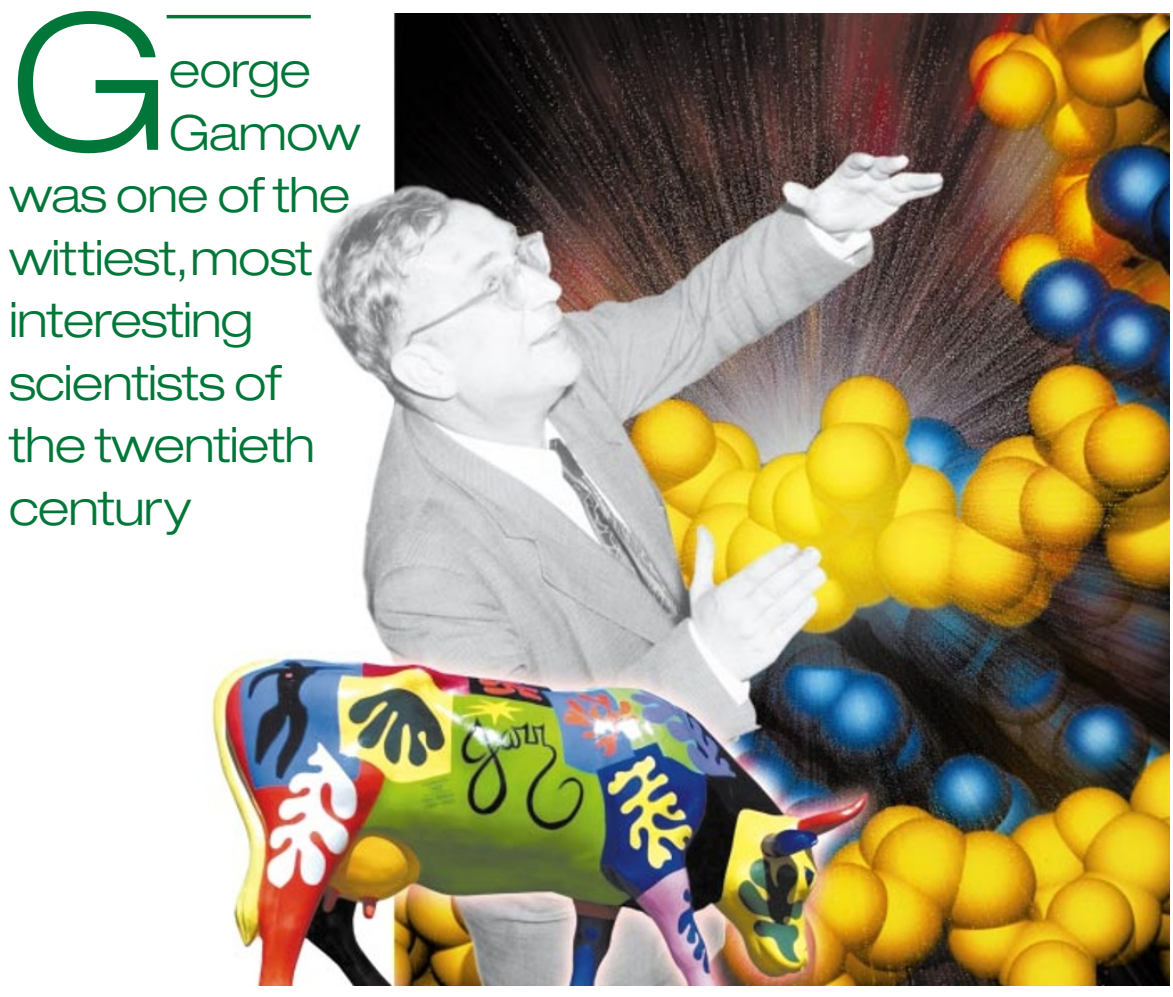

Practical jokes or profound ideas: Gamow considered a breathtaking range of scientific questions.

background that would be necessary to account for nuclear abundances, suggesting a residual five-degree background radiation.

We now realize that their scheme was incorrect. The Universe began with roughly equal numbers of protons and neutrons. Collisions with electrons, positrons, neutrinos and anti-neutrinos are more important than neutron decay, and the absence of stable nuclei with atomic numbers of five and eight creates a barrier to further fabrication in the early Universe. Nevertheless Alpher, Gamow and Herman's work was the first serious attempt to discuss the observable consequences of a big bang and the basic framework was correct. Ironically, the term 'Big Bang' was coined by Fred Hoyle, an advocate of a steady-state model of the universe, to make fun of Gamow's efforts.

In 1953, Gamow read the famous Watson and Crick Nature Letter on the structure of DNA and immediately jumped to the conclusion that the DNA molecule could directly serve as a template for protein synthesis. Recognizing that there are twenty independent combinations of triplets that can be made out of four letters, he suggested that there are twenty relevant amino acids and the existence of a one-to-one correspondence between triplets and amino acids, the backbones of proteins. In his autobiography, Crick remembers receiving a letter from Gamow suggesting this possibility, and then realizing that he and Watson had not even counted the number of amino acids. Crick, Gamow and Watson became good friends and Gamow even founded an RNA Tie Club, limited to twenty members, each one of whom had a special tie, provided by Gamow, with a specific amino acid in its design. The connection is not as direct as Gamow had envisioned: DNA makes RNA which makes amino acids and the code is not one-to-one, but Gamow's 1954 Nature Letter, "Possible relation between DNA and protein structures", was the first to appear on a subject which has changed our lives.

The DNA manuscript listed a Mr Tompkins as a co-author, but a vigilant editor removed his name. Gamow had written a series of popular books, in which a mythical MrTompkins explores the wonders of the world. Even without Mr Tompkins, Gamow was one of the wittiest, most interesting scientists of the twentieth century, a man with an extraordinarily quick and sure grasp of the great problems.

Gino Segrè is a professor of Astronomy and Physics at the University of Pennsylvania, 209 S. 33rd Street, Philadelphia, Pennsylvania 19104-6396, USA. 